\title{
A one-step method for taking plane hologram type holographic rainbow stereograms
}

\author{
Ming J. Huang a , C.K. Lee ${ }^{b}$, Ching-Piao $\mathrm{Hu}^{\mathrm{c}}$ \\ ${ }^{a}$ Department of Mechanical Engineering, National Chung-Hsing University, Taichung, Taiwan, R.O.C. \\ b Institute of Applied Mechanics, National Taiwan University, Taipei, Taiwan, R.O.C. \\ c National Science Council, 106 Ho-Ping E. Road, Section 2, Taipei, Taiwan, R.O.C. \\ Received 14 September 1994; revised version received 18 November 1994
}

\begin{abstract}
A new method for registering stereograms where orientated interference fringes provide the desired directivity for horizontal parallax is presented. With the help of a sirip shaped mask behind the ground glass, the stereograms obtained by this newly developed method produce a rainbow effect. In addition to having a wide angle of view, these stereo images can also be observed under an extended white light source. In addition, the plane hologram characteristics of the stereo images obtained by this method indicates that the image size will not scale with the variations of the reconstructing light source. A quantitative analysis on the distortion problems related to this method is discussed. The distortion-free conditions for making a holographic stereogram was developed using this theoretical analysis. A sensitivity analysis related to the viewing position errors for images recorded using this method is also presented.
\end{abstract}

\section{Introduction}

Holographic stereography was developed to circumvent the limitations imposed by conventional holography. Holographic stereography, the synthesis of photography and holography, provides a versatile means of displaying 3D images. Since the invention of the multiplex hologram (cylindrical holographic stereogram) by Cross [1], applications to such versatile fields as entertainment, education, advertisement, medicine, science, art, etc., have all been developed. Since 2D photographs are taken first, holographic stereograms do not have to record objects of interest directly holographically as required in conventional holography. The main drawbacks of the early developed cylindrical holographic stereograms lie in the the complicated optical setup needed to integrate the
2D photographs into 3D images. In addition, the finished hologram must be rolled into a cylindrical shape to generate the synthetic 3D images. In addition, the existence of the still unavoidable vertical lines on the finished hologram, and the unavoidable perspective \& time-smear distortions [2], and image blur [3] make one uncomfortable when viewing.

The flat format holographic stereogram, which not only is distortion-free and free of the annoying vertical lines but also is more convenient to display, was developed to overcome the problems mentioned above. In recent years, many methods that produce flat format holographic stereograms have been developed. By using a "fly's eye" lens to photo a 3D scene first and then projecting the recorded photograph through the same lens, 3D scene stereograms were made by Pole [4]. McCrickerd and George [5] recorded the objects' par- 
allax qualities in white light 1 y using a stereoscopic camera technique. DeBitetto |6] synthesized a composite hologram from a horiz ntal sequence of $n$ incoherently recorded two-dime isional photographs. In 1970 King et al. [7] developed a technique which uses only seconds of computer tim: and some automated optical manipulations to produ e extremely high quality holograms of computer-st red three-dimensional objects. Sopori and Chang [8] recorded every stereoscopic image on a slightly $\mathrm{d}$ fferent area along the holographic plate with differer $t$ angular directions. A new achromatic holographic ste reogram technique [9] was developed by Benton in 1781 . Rabal et al. [10] proposed a new method for reg stering stereograms in which an orientated speckle pittern supplies the desired directivity for obtaining corizontal parallax. In 1990 Kang et al. [11] introdused an image processing technique for making a oni-step flat format holographic stereogram in which hree-dimensional images can be observed at an arbit ary specified position.

A new and efficient one-step method that generates flat format holographic $3 \mathrm{D}$ scene stereograms is presented in this article. The holog'aphic stereograms obtained by this newly developerl method have a wide viewing angle and are observible under white light. Furthermore, due to its plane ho ogram characteristics, the rcconstructed stereo images are not scaled with the variations of the reconstructing light source.

\section{Theory}

This newly developed setup is illustrated in Fig. 1. The collimated beam modulatis by the photograph and thus carrying the image information is used as the object beam. The beam radiated from the ground glass is used as the reference beam. These interference fringes generated by the two beams are then recorded by the holographic filın. A double-exposure transmission hologram was ger erated from the stereo pair. During each exposure, either the right-half or the left-half of the illuminated areit of the ground glass was blocked. After the standard developing process, the hologram was then recons ructed by the conjugate object beam (plane wave). The recorded-images (the stereo pair) diffracted the reconstructing beam to the regions where the illuminated area of the ground glass originally occupied, respectively. By placing two eyes approximately in the position of the ground glass, one can see the two images (the stereo pair) autostereoscopically. The wide viewing angle of the reconstructed images are the effect of the ground glass. Since the beam through the ground glass is further restricted to a strip shaped area, the holographic stereograms produce a rainbow effect when reconstructed under white light. The sterograms generated by this newly developed setup can be viewed under any extended white light source. Both characteristics, wide viewing angle and observable under white light, make for a magnificent display.

The recorded images (the stereo pair images) are not scaled [12] even when the conditions of the reconstructing light sources change. This is due to the holographic stereogram recorded images generated by this newly developed method possessing plane hologram characteristics. It is assumed that the spatial frequency of the recorded photo image will not introduce a strong diffraction effect at the wavelength of the recording object beam. However, if the spatial frequency of the recorded photo image has too high a diffraction effect between the photo and the holographic plate, the photo should be placed in contact with the holographic plate in order to minimize the diffraction effect. As we know [13], from a spatial coherence point of view, the reconstructed image point shift distance $(\Delta r)_{\text {img }}$ is proportional to the reconstructing light source shift distance $(\Delta r)_{\mathrm{rsc}}$. That is,

$$
(\Delta r)_{\mathrm{img}}=\frac{z_{\mathrm{img}}}{z_{\mathrm{rsc}}}(\Delta r)_{\mathrm{rsc}},
$$

where $z_{\text {img }}$ and $z_{\text {rsc }}$ represent the vertical distance of the hologram with respect to the recorded images and the reconstructing light source, respectively. In the configuration shown in Fig. 1, $z_{\text {rsc }}$ approaches infinity and $z_{\mathrm{img}}=0$ for all values of $(\Delta r)_{\mathrm{rsc}}$, which is the typical plane hologram characteristics. From a temporal coherence point of view, the in-plane (a plane parallel to the holographic plate) and out-of-plane shift distance $(\Delta r)_{\text {img }}$ and $(\Delta z)_{\text {img }}$ of the reconstructed image point is proportional to the wavelength shift $\Delta \lambda$ between the reconstructing and recording light source and the vertical distance $z_{\mathrm{img}}$ of the recorded images from the hologram plane, i.e.,

$(\Delta r)_{\mathrm{img}}=\frac{r_{\mathrm{rsc}}}{z_{\mathrm{rsc}}} z_{\mathrm{img}}\left(\frac{\Delta \lambda}{\lambda}\right)$, 


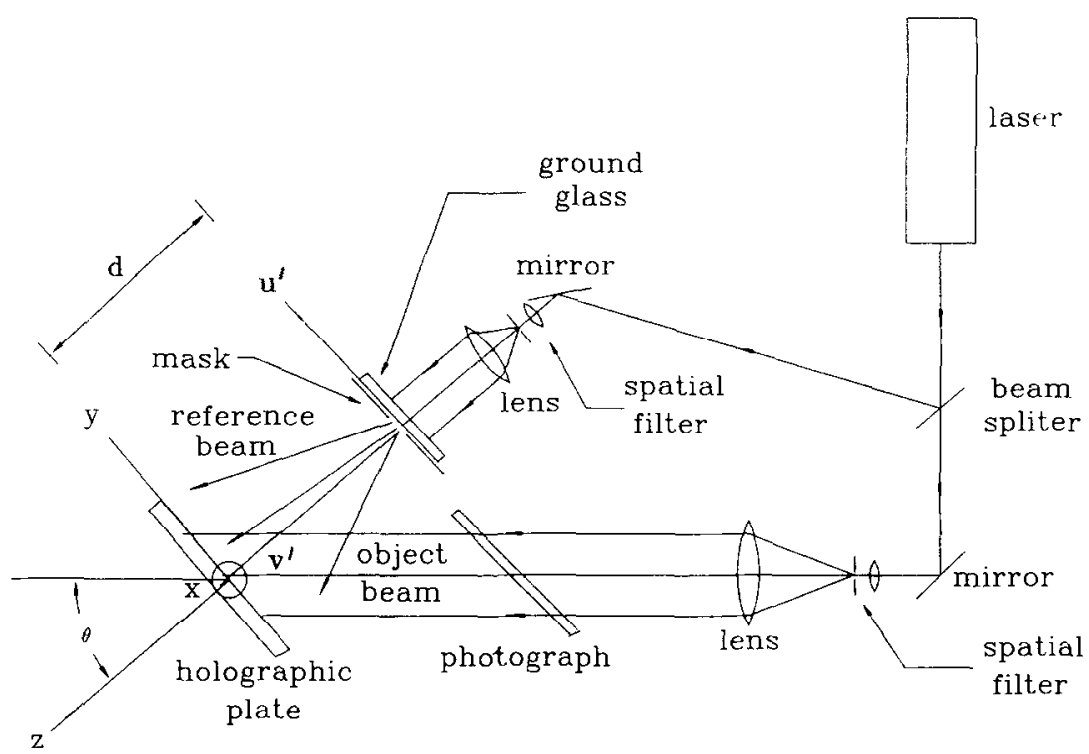

Fig. 1. Schematic drawing of the optical setup for obtaining "one-step plane hologram type holographic rainbow stereograms".

$(\Delta z)_{\mathrm{img}}=z_{\mathrm{img}}\left(\frac{\Delta \lambda}{\lambda}\right)$,

where $\lambda$ is the wavelength of the recording light source, $r_{\text {rsc }}$ and $z_{\text {rsc }}$ are the in-plane and out-of-plane distance between the reconstructing light source and the hologram plane, respectively. Again, $z_{\text {img }}$ is identically zero for the configuration shown in Fig. 1, $(\Delta r)_{\text {img }}$ and $(\Delta z)_{\text {img }}$ will always be zero, thus this newly developed method produces an "one-step plane hologram type holographic rainbow stereogram".

The ground glass introduced in Fig. 1 greatly improves the view-angle and the uniformity of the reconstructed images. In addition, the benefits of the optical configuration presented can be further understood by reversing the role of the reference beam and the object beam. That is, the mask and the ground glass can together be viewed as the image of the original object beam. From Eqs. (1) and (2), the variations of the wavelength and the size of the reconstructing light source, i.e., $\Delta \lambda$ and $(\Delta r)_{r s c}$, will only change the size of the reconstructed ground glass. Since the ground glass determines the view angle and serves as the illuminating light source during reconstruction, it is clear that the sterograms generated by this method will not change size no matter that $\Delta \lambda$ or $(\Delta r)_{\text {rsc }}$ varies.

Referring to Fig. 1, using $r(x, y)=R(x, y) \mathrm{e}^{-\mathrm{i} \phi(x, y)}$ to represent the light scattered on the hologram plane by the ground glass, and $a(x, y)=A(x, y) \mathrm{e}^{-\mathrm{i} 2 \pi \alpha y}$ to represent the light passing through the low spatial frequency photo then transmitted to the hologram plane, the intensity distribution of these two interfered beams is given by

$$
\begin{aligned}
& I(x, y)=|r(x, y)+a(x, y)|^{2} \\
& \quad=\left|R(x, y) \mathrm{e}^{-\mathrm{i} \phi(x, y)}+A(x, y) e^{-\mathrm{i} 2 \pi \alpha y}\right|^{2} \\
& \quad=R^{2}(x, y)+A^{2}(x, y) \\
& \quad+2 R(x, y) A(x, y) \cos [2 \pi \alpha y-\phi(x, y)],
\end{aligned}
$$

where $x$ and $y$ are the coordinates of the holographic plate, $R(x, y)$ and $A(x, y)$ are the non-negative amplitudes of the reference and object beam, respectively, $\phi(x, y)$ and $2 \pi \alpha y$ are the phases of the reference and object beam, respectively. The term $\alpha=\sin \theta / \lambda$ is a function of the light wavelength $\lambda$ as well as the angle $\theta$ between the object beam and the $z$ axis. Assuming a linear relationship between the amplitude transmittance of the developed hologram and the exposed intensity,

$t(x, y)=c_{1}+c_{2} I(x, y)$

where $c_{1}$ and $c_{2}$ are the constants determined by the holographic plate and the developing procedure. Since the fluctuation frequency of the $\cos [2 \pi \alpha y-\phi(x, y)]$ 


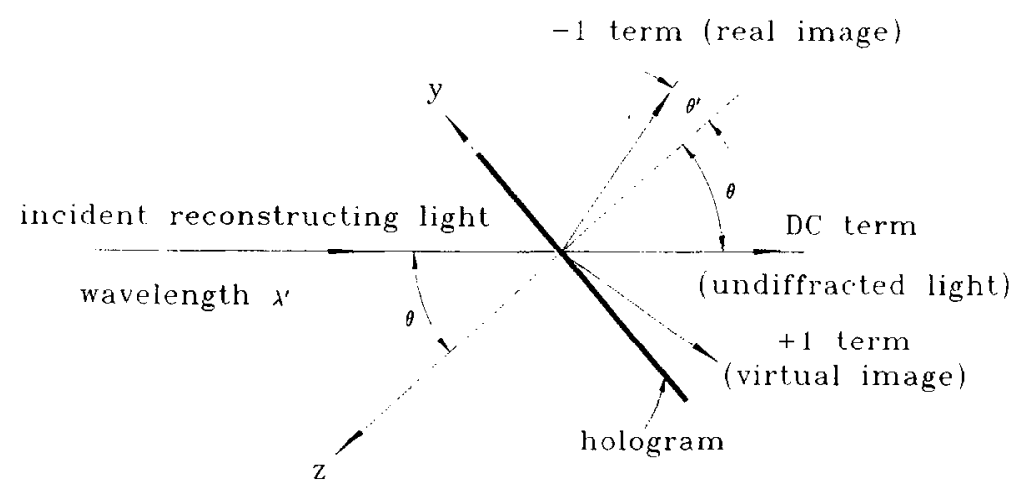

Fig. 2. The diffracted terms of the hologram recnnstructed by light beams with wavelength $\lambda^{\prime}$.

term is much higher than that of the $R^{2}(x, y)+$ $A^{2}(x, y)$ term, $t(x, y)$ can be fully characterized by $R(x, y) A(x, y) \cos [2 \pi \alpha y-\phi(x, y)]$, where $R(x, y)$ is almost uniform in the whole emulsion and $A(x, y)$ varies according to the $2 \mathrm{D}$ photo images. The larger the $A(x, y)$, the brighter the reconstructed images.

Reconstructing this hologram by the wave $B \mathrm{e}^{\mathrm{i} 2 \pi \beta y}$ (a plane wave with the same incident angle $\theta$ but a different wavelength $\lambda^{\prime}$ from the opposite direction of the object beam), the optical field $U(x, y)$ behind the hologram becomes

$$
\begin{aligned}
& U(x, y)=t(x, y) B \mathrm{e}^{\mathrm{i} 2 \pi \beta y} \\
& \quad=B c_{1} \mathrm{e}^{\mathrm{i} 2 \pi \beta y}+B\left[|r(x, y)|^{2}+A^{2}(x, y)\right] c_{2} \mathrm{e}^{\mathrm{i} 2 \pi \beta y} \\
& \quad+B c_{2} r(x, y) A(x, y) \mathrm{e}^{\mathrm{i} 2 \pi(\alpha+\beta) y} \\
& \quad+B c_{2} r^{*}(x, y) A(x, y) \mathrm{e}^{-\mathrm{i} 2 \pi(\alpha-\beta) y}
\end{aligned}
$$

where $B$ is a constant, $\beta=\sin \theta / \lambda^{\prime}$, and $\lambda^{\prime}$ is the wavelength of the reconstructing light.

The real image (Fig. 2 ) reconstructed by the conjugate wave of the incident light $r^{*}(x, y)$, which is represented by the last term of Eq. (5), is the only term of interest as it is observable on the ground glass during reconstruction. Thus, it is clear from Eq. (5) and Figs. 1 and 2 that the following condition must be met when $r^{*}(x, y)$ is used to reconstruct the stereogram

$\alpha-\beta=\frac{\sin \theta^{\prime}}{\lambda^{\prime}}$

where $\theta^{\prime}$ is the angle between the diffracted direction and the $z$ axis. If $S_{\lambda^{\prime}}$ represents the distance at the reconstructed ground glass between the images of the strip shaped area diffracted by $\lambda^{\prime}$ and $\lambda$, respectively, then

$S_{\lambda^{\prime}}=d \tan \theta^{\prime}$,

where $d$ is the distance from the hologram to the position of the reconstructed ground glass plane, now the position of the viewer, (Fig. 1). To avoid cross-talk between the two images reconstructed by light beams with wavelength $\lambda$ and $\lambda^{\prime}, S_{\lambda^{\prime}}$ must be larger than the height of the reconstructed ground glass image. For the case discussed above, the height of the reconstructed ground glass image approximatcly equals the height of the illuminated area of the original ground glass $h$. Combining the above discussions with Eqs. (6) and (7), and setting $\lambda^{\prime}=(1-c) \lambda$, the condition that guarantees the holographic stereograms generated by this newly developed configuration shown in Fig. 1 will not have any cross-talk during white light reconstruction becomes

$$
\frac{d c \sin \theta}{\sqrt{1-c^{2} \sin ^{2} \theta}}>h \text {. }
$$

\section{Distortion: problem and solution}

Photographing an object from two viewing angles and then creating a stereogram by the stereo pair, produces a stereoscopic effect which will not be completely the same as the original object [14]. For clarity, we will restrict the discussions to the $2 \mathrm{D}$ case here. Referring to Fig. 3, the two cameras, separated by $2 w$, face toward the same point $(0, p)$. In addition, the distance between the lens and the film of the camera is 


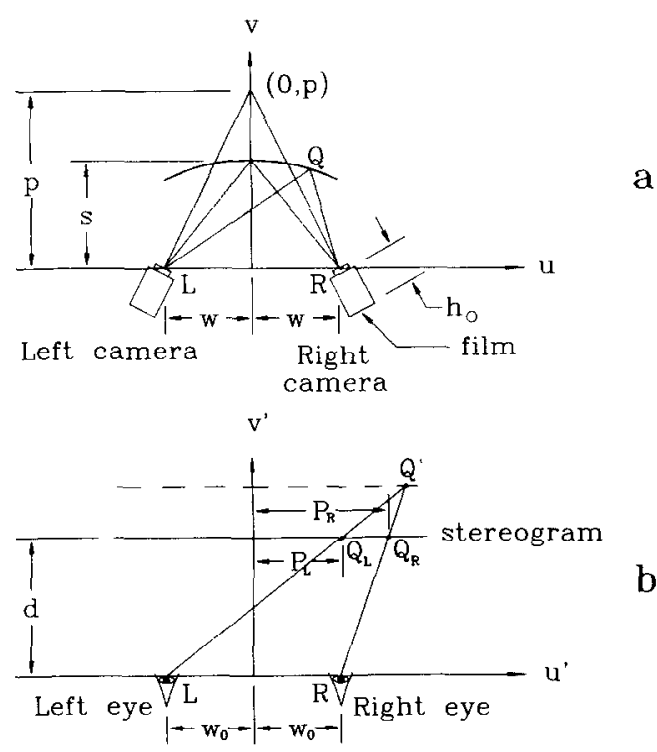

Fig. 3. The curve $f(u, v)=0$ will appear flat when viewed stereoscopically. (a) The geometry of taking the stereo pair of the curve $f(u, v)=0$. (b) The geometry of viewing the stereogram.

$h_{0}$, while the size of the stereogram image is $k$ times that of the photograph image (for the case shown in Fig. $1, k=1)$. In order for a set of images $f(u, v)=0$ on the 2D photos to appear as a line parallel to the stereogram, the distance between the two image points $Q_{\mathrm{R}}$ and $Q_{\mathrm{L}}$ on the stereogram, which correspond to the right and left images of the photographed object point, $Q$ must be a constant. More specifically, any point on $f(u, v)=0$ must satisfy (Fig. 3 )

$$
\begin{gathered}
k h_{0}\left[\tan \left(\cot ^{-1} \frac{w}{p}-\cot ^{-1} \frac{u+w}{v}\right)\right. \\
\left.-\tan \left(\cot ^{-1} \frac{-w}{p}-\cot ^{-1} \frac{u-w}{v}\right)\right] \\
=k h_{0}\left[\tan \left(\cot ^{-1} \frac{w}{p}-\cot ^{-1} \frac{w}{s}\right)\right. \\
\left.-\tan \left(\cot ^{-1} \frac{-w}{p}-\cot ^{-1} \frac{-w}{s}\right)\right] .
\end{gathered}
$$

Rewriting Eq. (9) yields a family of elliptical equations with major axis equaling to $s p+w^{2} / \sqrt{s p}$, minor axis equaling to $s p+w^{2} / p$ and centered at $\left(0, s p-w^{2} / 2 p\right)$ :

$s u^{2}+p\left(v-\frac{s p-w^{2}}{2 p}\right)^{2}=p\left(\frac{s p+w^{2}}{2 p}\right)^{2}$.

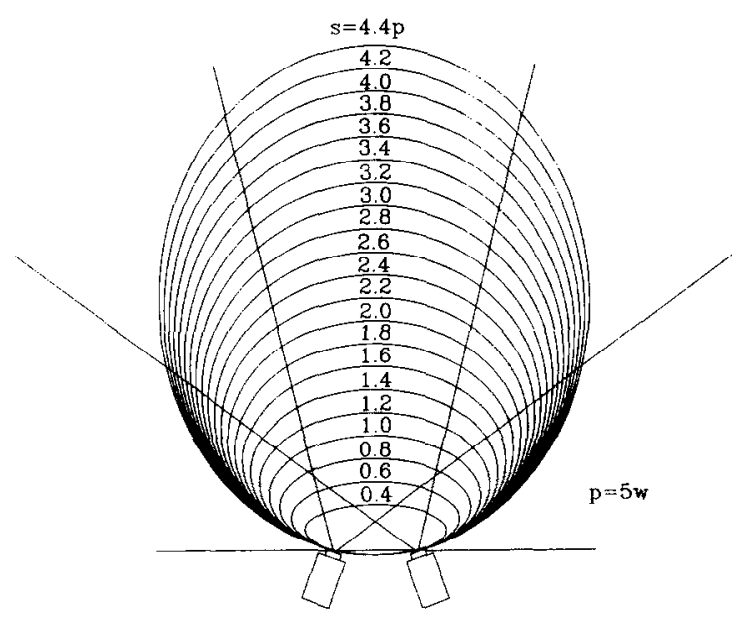

Fig. 4. The curves that will appear flat when viewed stereoscopically.

Some of these curves are shown in Fig. 4.

As shown in Fig. 4, the stereogram has less stereo distortion when the object is closer. This indicates that when the method presented in Fig. 1 is used to display the 3D stereo effect, placing the photographed object closer to camera is certainly one way of avoiding a large distortion. However, increasing the stereoscopic effect by simply placing the camera closer to the object possesses some major drawbacks. The large distance between $Q_{\mathrm{R}}$ and $Q_{\mathrm{L}}$ on the stereogram will make the viewing uncomfortable and thus difficult to combine these two images into a 3D sensational image through use of visual perception.

Eq. (10) suggests that a flat object will generally be distorted to become convex shaped provided no other special conditions are met. This observation can be further quantified. Considering a stereogram made from a stereo pair of a flat object whose surface is located at $v=s$ (Fig. 5), and is viewed from a $u^{\prime}$ axis with a distance $d$ from the stereogram, the location of the object point $Q$ appears at $Q^{\prime}\left(u^{\prime}, v^{\prime}\right)$ by visual perception. In addition, the geometrical analysis for the condition mentioned yields

$v^{\prime}=\frac{2 w_{0} d}{2 w_{0}-P_{\mathrm{R}}+P_{\mathrm{L}}}$,

$u^{\prime}=\frac{v^{\prime}}{2 d}\left(P_{\mathrm{R}}+P_{\mathrm{L}}\right)$,

where 

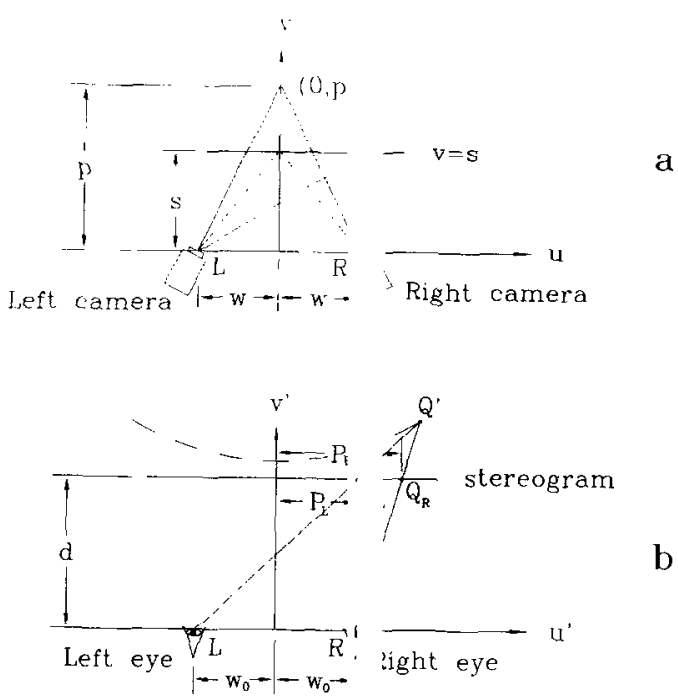

b

Fig. 5. The line $v=s$ will apper 1 onvex when viewed stereoscopically. (a) The geometry of $t: 1$ ig the stereo pair of the line $v=s$. (b) The geometry of viewil। the stereogram.

$P_{\mathrm{R}}=k h_{0} \tan \left(\cot ^{-1} \frac{-w}{p}-\frac{-1}{s-w}\right)$,

$P_{\mathrm{L}}=k h_{0} \tan \left(\cot ^{-1} \frac{w}{p}-\operatorname{co} \frac{u+w}{s}\right)$.

Again $2 w$ is the distance $b$ : veen the two cameras, $2 w_{0}$ is the distance between 1$\}$ two eyes of the viewer, $k$ is the size of the stereo: im image over that of the photograph image, and $l^{\prime}$ s the $v$-axis distance of the facing point of the two : meras from the origin. Substituting Eqs. (12) into | |s. (11), we obtain

$$
\begin{aligned}
v^{\prime} & =2 w_{0} d\left(2 w_{0}\right. \\
& \left.+2 k h_{0} w \frac{\left(w^{2}+s p\right)(p-}{\left(w^{2}+s p\right)^{2}-} \cdot \frac{1-p u^{2}}{w u)^{2}}\right)^{-1}, \\
u^{\prime} & =\left[k h_{0} s u\left(w^{2}+p^{2}\right)\right]\left\{\left(w^{\prime}-s p\right)^{2}-(w u)^{2}\right. \\
& +k h_{0}\left[\left(w^{2}+s p\right)\left(p-s \mid-p u^{2}\right]\right\}^{-1} .
\end{aligned}
$$

Under the parameters of ou: :xperiment $u^{\prime}$ is almost equal to $u$, therefore the stere: scopic distortion can be examined by plotting $v^{\prime}$ ver: $\mathrm{s} u$ as shown in Fig. 6 . From these curves, we see $t$ it a flat object becomes convex.

The distortion problems aused by synthesizing different perspective $2 \mathrm{D}$ ph ographs into 3D holo-

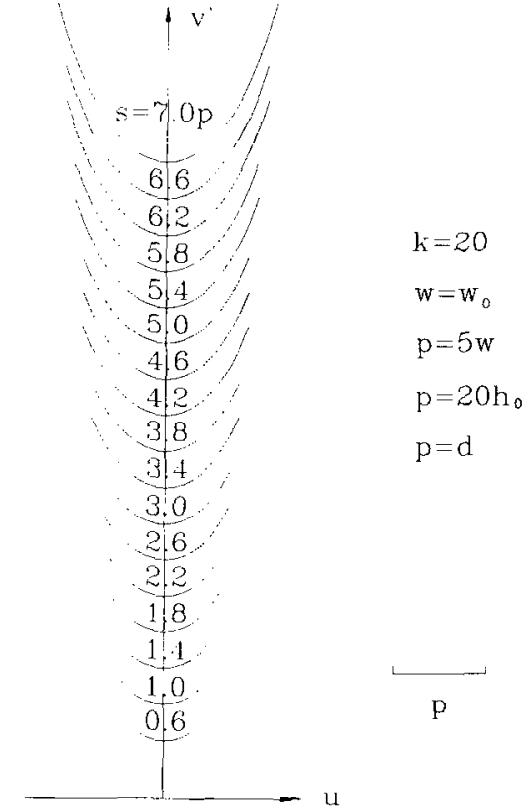

Fig. 6. Straight lines appear when viewed stereoscopically.

graphic stereogram images are so serious that have to be resolved in order to offer distortion-free images for applications in fields such as metrology, medical or education. To resolve this problem fully, we can shift the images point by point based on Eqs. (11)-(13), respectively. Despite the fact that this compensation can be done by using software automatically, it is still extremely cumbersome and certainly impractical. A simple hardware compensative method will be discussed here based on the distortion analysis presented above. It is clear from Eq. (9) that as the $p$ value approaches infinity, i.e., each camera pointing straight forward, the curve $f(u, v)=0$ reduces to a line $v=s$. This result shows that a line remains a line after this specific synthesizing and viewing process. There is no distortion problem appears in this condition. More specifically, by taking the $2 \mathrm{D}$ perspective images with $p$ approaching infinity (see Fig. 3a), we can generate distortion-free holographic stereograms.

The distortion-free condition, i.e., setting $p$ approaching infinity, can be achieved experimentally by the procedures shown in Fig. 7. Firstly, one can record the $2 \mathrm{D}$ perspective photos with the two cameras facing straight toward the object of interest (Fig. $7 a)$. The intersection of the line-of-sight of the two cameras with the objects are denoted as $R_{\text {img }}$ and 

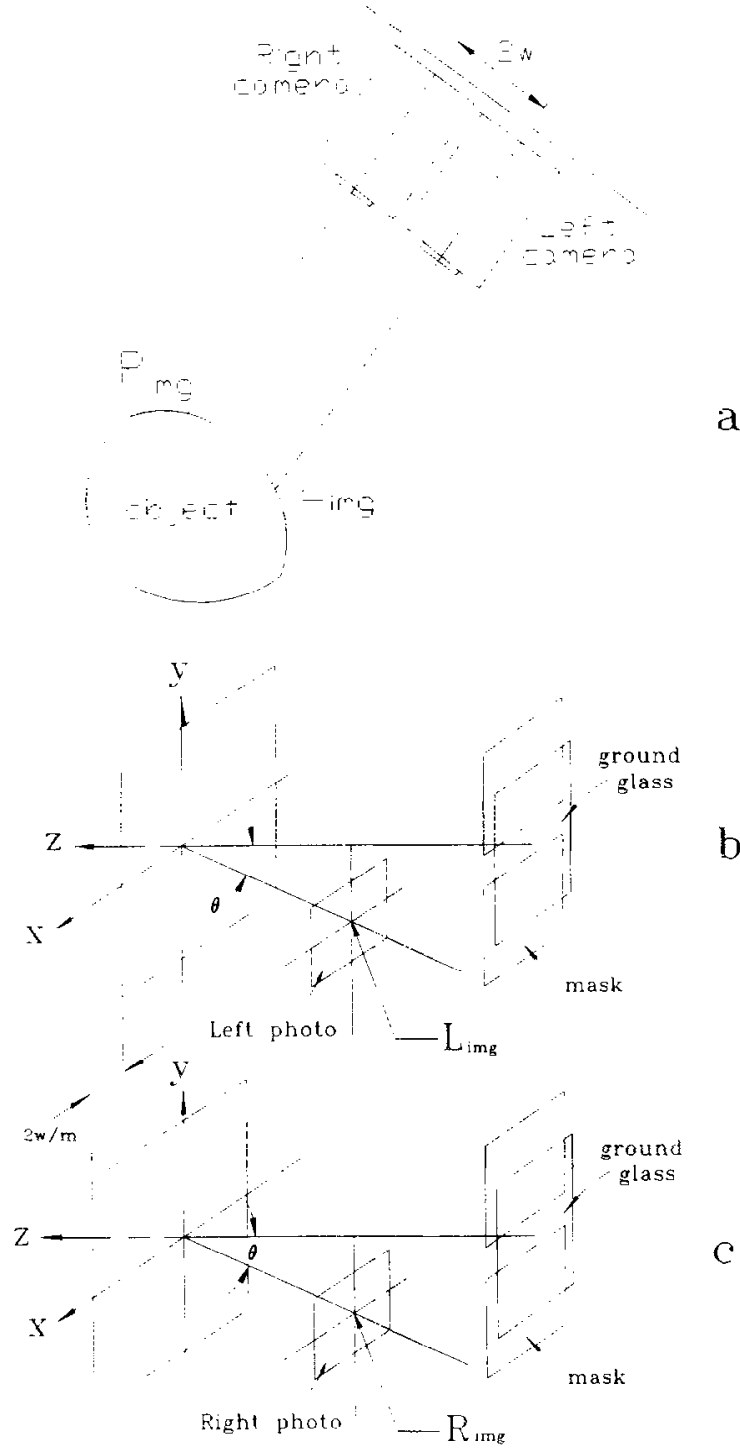

Fig. 7. Procedures for making a distortion-free image hologram. (a) recording the $2 \mathrm{D}$ perspective image, (b) synthesis of the left perspective image, and (c) synthesis of the right perspective image.

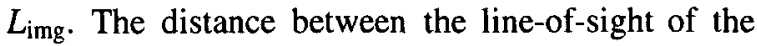
two cameras is $2 w$. Secondly, the photos taken by the left camera is recorded holographically onto the holographic stereogram with the configuration shown in Fig. 7b. Finally, the photos taken by the left camera is replaced by the photos generated by the right camera, with care given to ensure that the placement location of the two photos are identical. Since we reconstruct the image by using the conjugate plane wave (Fig. 2) and to ensure the two perspective angles between recording and synthesis are identical, the hologram has to be shifted in-plane by a distance equaling to $2 w / m$, where $m$ is the magnification ratio between the object and the 2D perspective image. More specifically, the perspective angles between the object of interest and the 2D perspective photos as well as the perspective angles between the holographic stereogram and the viewer are preserved by this procedure [15]. One thing that should be noted is that this distortion-free condition can be equally applied to many different configurations of synthesizing holographic stereograms. For example, this condition can be achieved experimentally by using exactly the same procedure for both the method proposed in this article as well as for the method proposed by DeBitetto [6]. After each exposure, instead of shifting the mask proposed by DeBitetto [6], the distortion-free condition can be achieved by shifting the hologram one slit distance before taking the next frame of images as was described above.

It should be noted that even though $k$ is equal to one for the case shown in Fig. 1, the derivation of the distortion-free condition presented above (Eqs. (9)(13)) does not hinge on the value of $k$ to be 1 , i.e., the distortion-free condition presented is applicable to $k$ of any value. This understanding leads to at least two ways of making large format holographic stereograms. The first method performs the magnification at the steps of photographing the objects and then uses the same configuration shown in Fig. 1 to make the holographic stereogram. In the case, where $k=$ 1 , the distortion-free application is certainly be valid. The second method magnifies the photo images onto the holographic plate during the synthesizing stage by making $k \neq 1$. For example by inserting a low aberration beam expander between the photo and the holographic plate in Fig. 1, this condition can be achieved without any derivation from the fundamental thinking of the distortion-free condition presented. Certainly, by combining the two methods discussed here, an even larger magnification ratio can be achieved.

In the above discussion, we assumed that viewers are standing at the designated viewing positions without any errors. At this designated viewing position, the $3 \mathrm{D}$ images synthesized at the distortion-free condition mentioned above will not distort. However, view- 

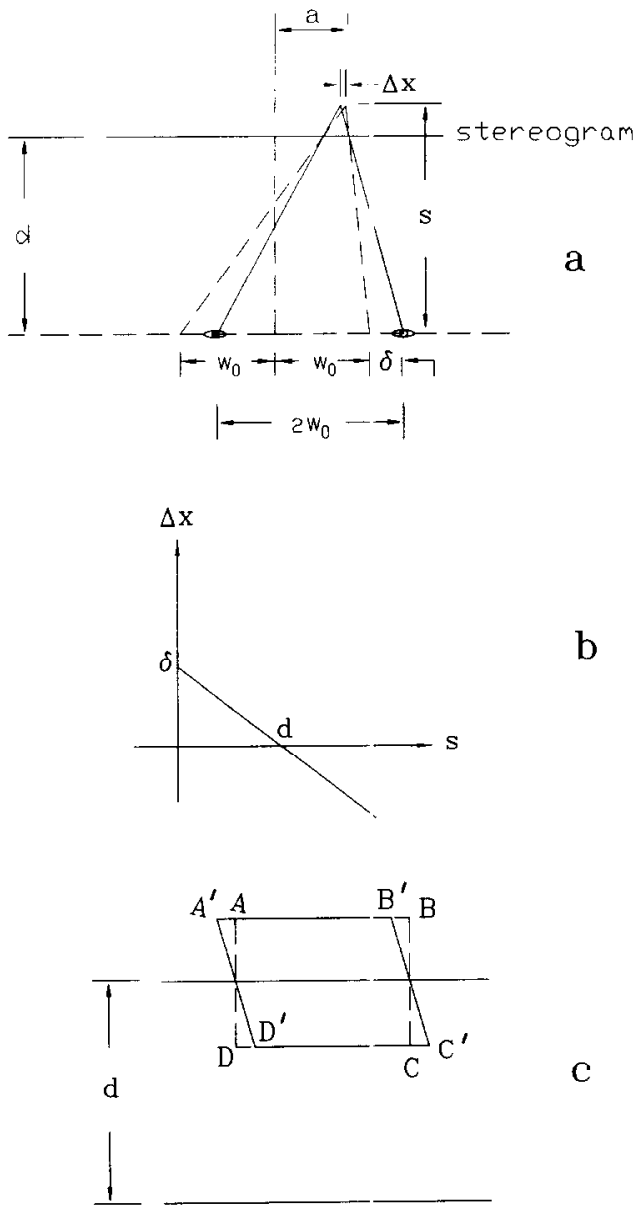

Fig. 8. (a) The geometry of the horizontal viewing position error. (b) The relationship between $\Delta x$ and $s$. (c) The shape distortion caused by a horizontal position error.

ers can seldom position themselves at the exact position to view the holographic stereogram, therefore, the synthesized 3D images will still distort even for the distortion-free condition mentioned above. The sensitivity between viewing the position error and the distortion will be discussed next to gain some insight into designating the positioning of the synthesized 3D images.

Referring to Fig. 8a, if the viewer's position misses a horizontal distance $\delta$ the synthesized image point will shift in the horizontal dircetion by a distance

$\Delta x=\frac{d-s}{d} \delta$,

where $d$ is the vertical distance between the holographic stereogram and the viewer and $s$ is the verti- cal distance between the original image point and the viewer. Eq. (14) shows a linear relation between the image horizontal shift distance $\Delta x$ and the viewer's position error $\delta$. The vertical distance $s$ of the image point will influence the image shift as well. The larger the image point away from the holographic stereogram plane, the more the synthesized 3D image point shift. The relationship between $\Delta x$ and $s$ are shown in Fig. $8 \mathrm{~b}$. It is clear that the horizontal viewing position miss will cause the rectangle $A B C D$ to be deformed into a parallelogram $\mathrm{A}^{\prime} \mathrm{B}^{\prime} \mathrm{C}^{\prime} \mathrm{D}^{\prime}$ (Fig. 8c).

If the viewing position is missed by an angle $\Delta \theta$ (Fig. 9a) instead of the horizontal positioning error mentioned above, the synthesized image point will shift both a horizontal and transverse distance $\Delta x$ and $\Delta z$, respectively. In practical applications, the angular viewing position error will not be a large number, therefore, this allows us to use a simple geometric analysis to show that the distortion along the $x$ and $z$ axes are

$$
\begin{aligned}
\Delta x & =\frac{\left(a^{2}-w_{0}^{2}\right) \Delta \theta}{d+a \Delta \theta} \frac{d-s}{s}, \\
\Delta z & =w_{0} \Delta \theta-s+\left(\frac{d a+w_{0}^{2} \Delta \theta}{d+a \Delta \theta}\right. \\
& \left.+\frac{\left(a^{2}-w_{0}^{2}\right) d \Delta \theta}{s(d+a \Delta \theta)}+w_{0}\right) \frac{s\left(d-w_{0} \Delta \theta\right)}{d\left(a+w_{0}\right)},
\end{aligned}
$$

where $a$ is the $x$-coordinate of the discussed image point. It is clear from Eqs. (15) that the relationship between the image point shift distance and the angular viewing angle error is non-linear. For the case $d \gg a \Delta \theta$, where $1 /(d+a \Delta \theta) \approx(1-a \Delta \theta) / d$, Eqs. $(15 \mathrm{a}, \mathrm{b})$ reduce to

$\Delta x \approx \frac{\left(a^{2}-w_{0}^{2}\right)}{s} \frac{d-s}{d} \Delta \theta$,

$\Delta z \approx \frac{d-s}{d} a \Delta \theta$

It is clear from Eq. (16) that for some constant s, $\Delta x$ is a parabolic function of $a$ and $\Delta z$ is a linear function of $a$. A horizontal shift $\Delta x$ and a transverse shift $\Delta z$ will cause a rectangle to be deformed into a geometric shape as shown in Figs. $9 \mathrm{~b}$ and $9 \mathrm{c}$. By combining these two-dimensional effects, a rectangle will be deformed into a shape like Fig. 9d. 


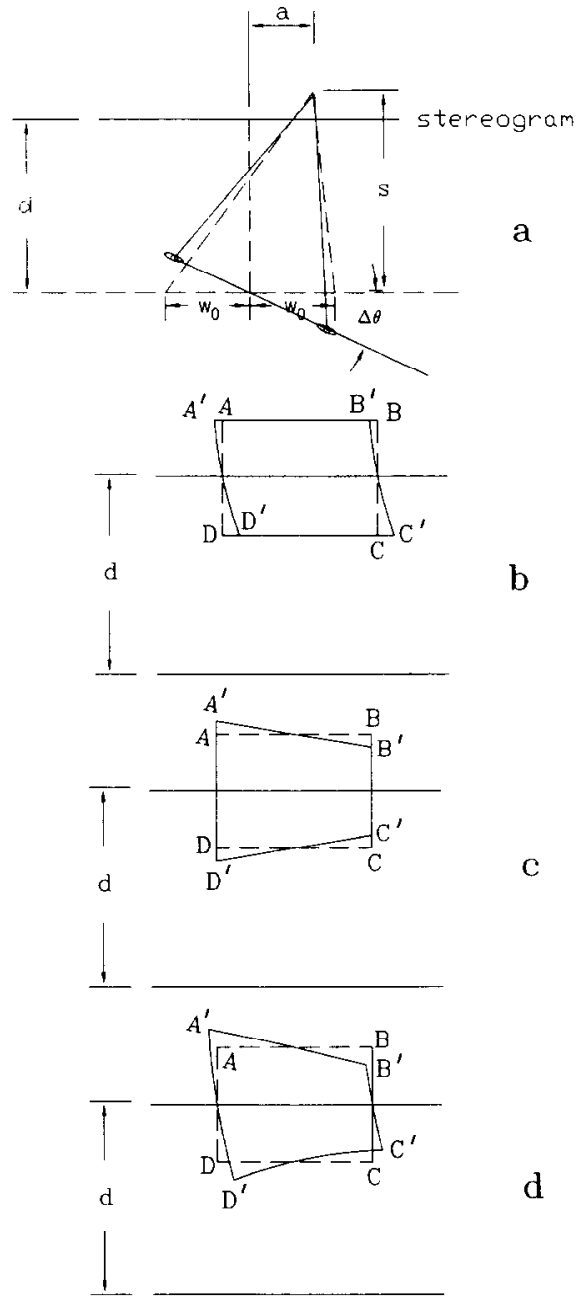

Fig. 9. (a) The geometry of an angular viewing position miss. (b), (c) The horizontal and transverse shift caused by an angular viewing position miss. (d) The shape distortion caused by an angular viewing position miss.

\section{Experiments and results}

First, two photographs taken by two cameras separated by a certain distance, of a 3D object (in this experiment, a dinosaur model) were obtained under white light. Then adopting the distortion-free procedures (Fig. 7) on the setup similar to Fig. 1, two registers of this scene were exposed to the same photosensitive medium (in the case presented here, Agfa 8E75 film was used). The photograph was placed parallel to the photosensitive emulsion and inclined with the collimating beam such that the reconstructed stereo- gram images have the same size as the original photograph. A HeNe laser with $\lambda=632.8 \mathrm{~nm}$ was used as the light source, a pupil $2.3 \mathrm{~cm}$ in height and 10 $\mathrm{cm}$ in width was used as a mask to block the ground glass. The distance between the ground glass and the holographic plate was $45 \mathrm{~cm}$, the distance between the $2 \mathrm{D}$ photo plane and the holographic plate was 10 $\mathrm{cm}$, and the hologram-recorded-images were within a $5 \mathrm{~cm} \times 5 \mathrm{~cm}$ area. The angle between the object beam and the reference beam $\theta$ equaled $30^{\circ}$. For the parameters used in this experiment, $h$ shown in Eq. (8) was limited to less than $45 \mathrm{~mm}$ to avoid cross-talk of the ground glass images reconstructed by the different wavelengths when $c$ is chosen to be 0.2 .

It is well-known that the speckle noise is proportional to $\lambda q / D$, where $\lambda$ is the light beam wavelength, $\mathrm{q}$ is the distance between the scattering surface to the image plane, and $D$ is the aperature size. In our experiment the dominant aperature size is the smaller of the height $h$ and the effective width $l / n$ of the slit, where $l$ is the width of the slit and $n$ is the number of perspective angles used in the synthesizing step. In our experiment $h=2.3 \mathrm{~cm}, l=10 \mathrm{~cm}$ and $n=2$, the dominant aperature size is the height of the slit. The speckle noise is experimentally verified to be acceptable. However, it should be noted that if we intend to increase the perspective angles using this one-step holographic stereogram, the value of $n$ must be increased with care so as not to make the speckle noise deteriorate to an unacceptable condition. The traditional two-step holographic stereogram places the slit during the making of the so called H1-hologram [6]. More specifically, the second step of the traditional two-step holographic stereogram reconstructs the real image of the photo onto the hologram plate through the same distance (as that of $d$ shown in Fig. 1), i.e., the speckle noise of traditional two-step holographic stereogram went through the distance $d$ twice. Since speckle noise is generated by the incoherent sum of the input laser beam, the phase reconstruction process of the traditional two-step holographic stereogram will not nullify the speckle noise. In contrast, the speckle noise introduced within this newly developed one-step holographic stereogram is propagated through the distance $d$ only once. Therefore the speckle noise problem is at least in theory less severe in this newly developed method than that of the two-step holographic stereogram. The dominant noise in the making of holo- 

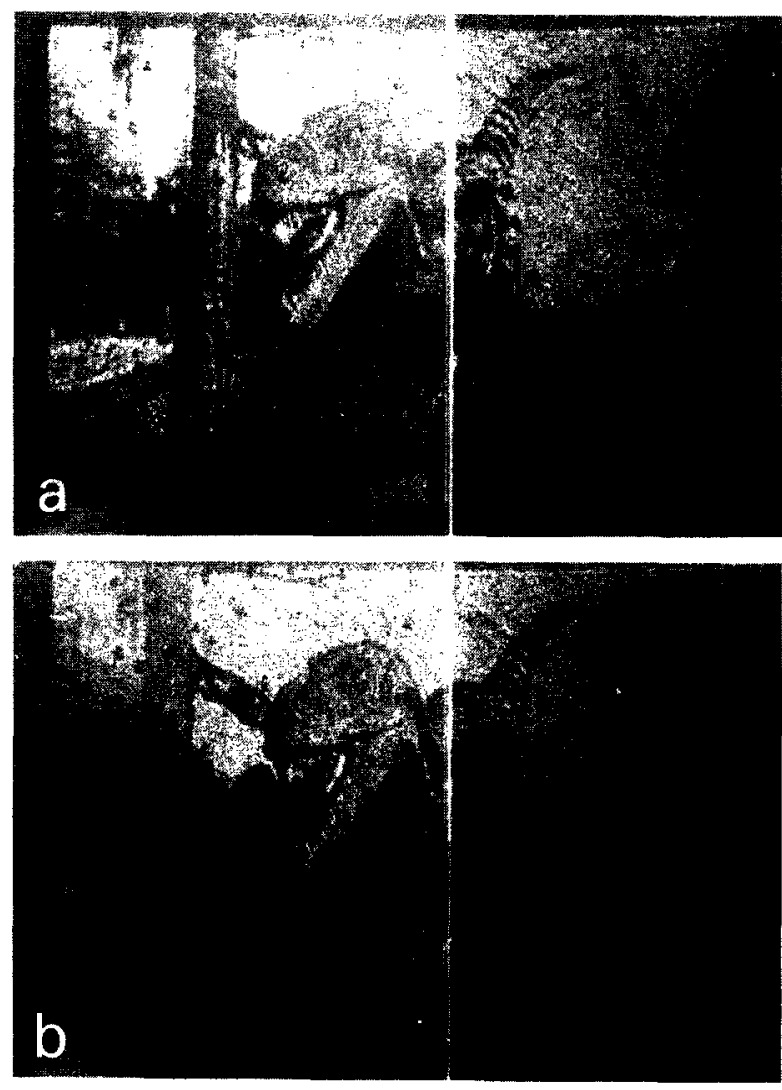

Fig. 10. Photographs of the reconstructed image taken from (a) left. and (b) right viewing positions.

graphic stereogram is by no means the speckle noise as the efficiency of the holographic stereogram was well understood to be inversely proportional to the square of the numbers of the synthesizing steps. The reduction in diffraction efficiency will quickly over power the speckle noise condition when the number of perspective angles are increased.

A standard bleaching procedure was applied to the hologram developed and found to increase the efficiency markedly. In Fig. 10, the two images reconstructed from the stereogram corresponding to the two different points of view of this dinosaur model are shown. These two images: corresponding to two slightly different points of view of the dinosaur model, when viewed under each associated eye, will create a 3D image of the dinosaur alter visual perceptionis processed by the brain.

\section{Conclusion and discussion}

A new one-step method for generating holographic stereograms has been successfully developed. The holographic stereograms obtained by this new method has a wide angle of view and appears colorful under white light. Furthermore, the reconstructed stereo images are not scaled with the variations of the reconstructing light source. A distortion-free condition applicable to many different methods of generating holographic stereograms was also presented in detail. An experimentally feasible approach to arrive at this distortion-free condition was discussed also. After arriving at the distortion-free condition in the synthesis stage, the image distortion effect during the observation stage was also evaluated quantitatively to facilitate the placement of the holographic stereogram at different exhibition conditions.

The results presented in this article can also be obtained by placing the recorded photograph directly in contact with the photosensitive emulsion and registering twice by replacing the photograph during each exposure. In comparison, the non-contact method shown in Fig. 1 is more convenient and can avoid scratching the emulsion. More importantly, we can replace the photograph by the LCD (liquid crystal display), which can be connected with a CCD camera or computer to generate the input images, to make the input more flexible and versatile.

The stereoscopic effect can be enhanced by increasing the distance between the stereo pair, and the resulting 3D image can be located behind or in front of the registered plate by choosing a point in the scene and putting it in coincidence with its homologous in double exposure. This of course will introduce some distortion and change the stereoscopic effect of the reconstructed image. The selected point appears to be lying on the plane of the stereogram and all others appear to be behind or in front of that plane, which is in accordance with their relative position in the object.

Till now we have restricted our input only to stereo pairs. However, this is not the only way to generate a stereogram. We can use three, four, or more photographs (the exact stereoscopic views of the recorded object) as the input images, and registering with the light radiated from the corresponding area of the ground glass, respectively. This result will cause multiple exposures of the hologram. As we know, the 
more times the hologram is registered, the more continuous the stereogram appears to be, but the lower the efficiency.

Combining this method with embossed holography will make mass production of this easily achievable.

\section{References}

[1] L. Cross, SPIE, San Diego, CA, USA, Aug. (1973).

[2] S.A. Benton, J. Opt. Soc. Am. 68 (1978) 1440.

[3] K. Okada, T. Honda and J. Tsujiuchi, Optics Comm. 41 (1982) 397.

[4] R.V. Pole, Appl. Phys. Lett. 10 (1967) 20.

[5] J.T. McCrickerd and N. George, Appl. Phys. Lett. 12 (1968) 10.
[6] D.J. DeBitetto, Appl. Optics 8 (1969) 1740.

[7] M.C. King, A.M. Noll and D.H. Berry, Appl. Optics 9 (1970) 471.

[8] B.L. Sopori and S.C. Chang, Appl. Optics 10 (1971) 2789

[9] S.A. Benton, J. Opt. Soc. Am. 71 (1981) 1568.

[10] H.J. Rabal, E.E. Sicre, N. Bolognini, R. Arizaga and M. Garavaglia, Appl. Optics 22 (1983) 881.

[11] D.K. Kang, M. Yamaguchi, T. Honda and N. Ohyama, Optics Comm. 80 (1990) 7.

[12] A. Olivares-Perez, L.R. Berriel-Valdos and A.A. Morales, Appl. Optics 28 (1989) 4366.

[13] R.J. Collier, C.B. Burckhardt and L.H. Lin, Optical Holography (1983)

[14] D.B. Diner, Appl. Optics 28 (1989) 1737.

[15] M.J. Huang, Ph.D. Dissertaion, Dept. Mech. Eng.. National Taiwan Univ. (1994) 33. 